\title{
One killer virus, three key questions
}

\section{As the world mobilizes against the $\mathrm{H} 1 \mathrm{~N} 1$ flu pandemic, researchers are working to answer pressing questions about the virus. Brendan Maher visited pathologists at the US Centers for Disease Control and Prevention who are looking at how the virus kills, and a New York laboratory that is testing how it spreads. Declan Butler spent time at a French biosafety level-4 facility where researchers are working out the chances that the pandemic virus will reassort with the H5N1 avian flu virus.}

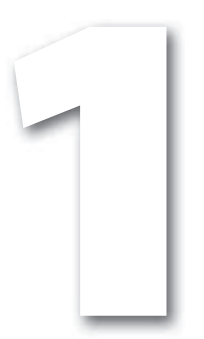

\section{How does it kill?}

There are five sets of eyepieces on the microscope, and Sherif Zaki is looking down one of them. Looking down the other four are members of the team he leads at the infectious-disease pathology branch at the Centers for Disease Control and Prevention (CDC) in Atlanta, Georgia. Other researchers trained in epidemiology, microbiology and electron microscopy watch a large, flat-screen monitor at the end of the narrow conference room that shows an image projected from the microscope.

Dianna Blau, an epidemiologist, reveals the source of the tissue: an 11-year-old girl who died in September, probably from $\mathrm{H} 1 \mathrm{~N} 1$ influenza. The team had already detected viral RNA in the girl's samples. But observing the presence of the H1N1 virus, especially in this tissue from deep down in the lungs, provides a more accurate and detailed diagnosis. The tissue seems messy, and is flooded with blue-stained Staphylococcus aureus. Zaki scans back and forth across the slide, seeking the hint of red staining that would indicate the presence of antibodies bound to the H1N1 virus. $\mathrm{He}$ finds it on one slide, where a red blob indicates a profusion of virus being released from a rupturing cell. They mark her as positive.

Zaki's lab holds these 'sign out' meetings every afternoon, examining autopsy and other tissues that have been sent to the $\mathrm{CDC}$ from doctors and medical examiners around the world to find, confirm or rule out any number of diseases. Since April, their case load has doubled thanks to H1N1. So far, the group has received samples from more than 300 suspect cases, and it has confirmed swine flu in more than a third of them.

In addition to providing a diagnosis, the group's analyses are helping to build up a picture of how the virus kills. Pathological studies can show what tissues are affected and to what extent, adding detail to the worldwide monitoring and surveillance efforts.

The profile emerging is of a distinctive virus. Although seasonal flu tends to infect just the cells high in the upper airway, H1N1 penetrates down into the terminal air sacs called alveoli. "This is not an area of the lung where you would usually see seasonal flu," Zaki says. He has seen such behaviour before, though - in the few samples of lung tissue he has examined from humans killed by the $\mathrm{H} 5 \mathrm{~N} 1$ avian flu virus. But the virus is much more prevalent in the tissues from the severe H1N1 cases he has examined — "like avian flu on steroids" as Zaki puts it.

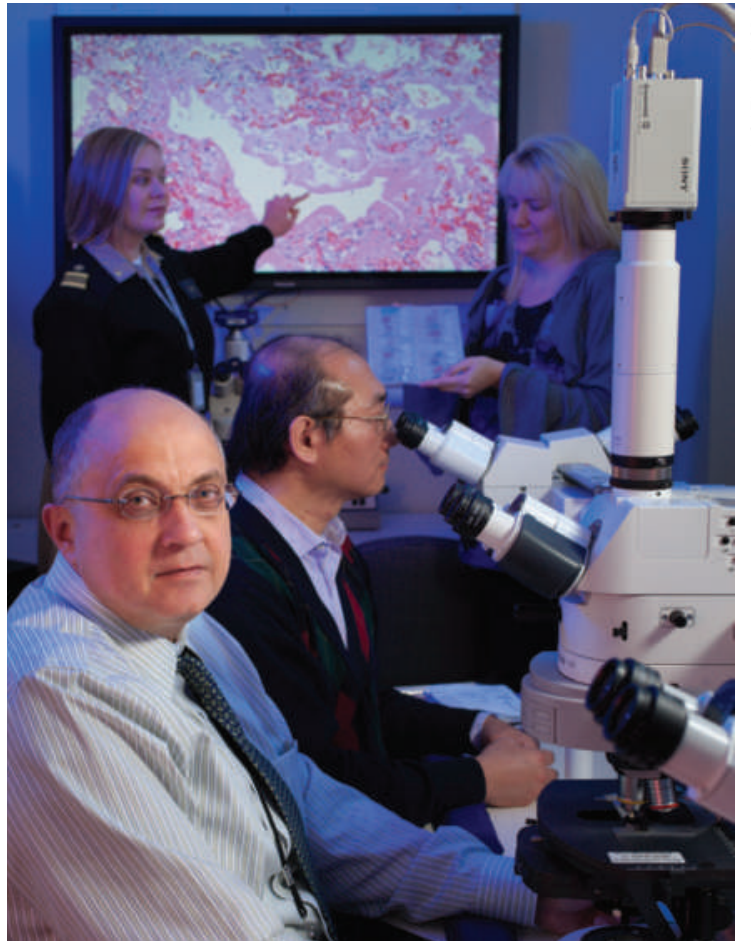

Sherif Zaki (front) studies tissue infected by pandemic H1N1.

Zaki says that his observations fit well with recent research looking at the mechanism of infection. A group led by Mikhail Matrosovich at Philipps University Marburg in Germany and Ten Feizi at Imperial College London studied sialyl glycans, glycoproteins that the flu virus binds to in order to gain entry to human cells ${ }^{1}$. Although seasonal strains of $\mathrm{H} 1 \mathrm{~N} 1$ bind mostly to versions of the glycoproteins known as $\alpha 2-6$, the researchers found that the new pandemic $\mathrm{H} 1 \mathrm{~N} 1$ can also bind to a version called a2-3, which is found in greater proportion in the lower respiratory tract.

Co-infection is common with pandemic $\mathrm{H} 1 \mathrm{~N} 1$, at least in those who have died. Zaki's group has observed infection with bacteria such as S. aureus or Streptococcus pneumoniae in about a third of the fatal swine-flu cases it has examined. In the rest, the virus seems to be lethal on its own. Zaki slots onto the microscope stage a slide from a 38-year-old male who died without bacterial co-infection. This one is filled with the red staining; also the walls of the alveoli are ruptured and blood cells and fluid fill the spaces normally 
reserved for gas exchange. Scar-like pink ribbons, called hyaline membranes, arc through the tissue. Pathologists call this state 'diffuse alveolar damage', and it tells them that the man had respiratory distress syndrome. "It's very difficult to treat a patient once they get to this state," Zaki says. He points out that the man was obese and had a history of hypertension and heart problems. Some $90 \%$ of the cases his team has reviewed have had some underlying medical condition.

Zaki says that they plan to publish some of their observations soon, and he has been sharing them with others at the CDC and with the public-health community at large. Although pathology can't predict what the virus will do in

"H1N1 looks
like avian flu
on steroids."
- Sherif Zaki
tions," she says, explaining that pathology results have led the CDC to recommend pneumococcal vaccination for people in at-risk groups.

At the end of the sign-out meeting, Zaki walks back to his office through a quiet lab; the technicians have gone home for the day and the machines used to prepare and stain slides are silent. Six fresh case studies in purple folders sit in a neat row along one of the lab benches ready for processing and examination. They contain slides or tissue embedded in paraffin: one still has lumps of formalin-soaked flesh sitting in a plastic specimen jar.

As the flu season ramps up, Zaki says, he suspects the number of flu cases will increase the future, it can help to identify those most at risk of severe disease. "We've really learned a lot with the lab pathology," says Anne Schuchat, director of the CDC's National Center for Immunization and Respiratory Diseases. "Both the prominence of the pneumonia in some infections and bacterial co-infection have important clinical implicadramatically. Still, his lab sees only a small slice of what's happening worldwide. "We're looking at the tip of the iceberg in terms of these cases," he says. It's a grim job, reading the tales of the dead, but he adds "that information is important for the living".

\section{Brendan Maher}

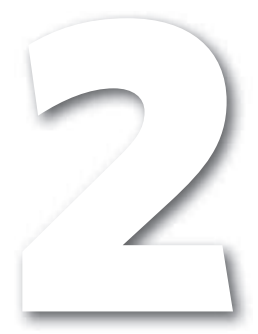

\section{How does it spread?}

In a cramped locker room high above the streets of upper Manhattan, laboratory personnel take a visiting journalist into the emerging pathogens facility, an enhanced biosafety level 3 (BSL-3) laboratory on the New York campus of Mount Sinai School of Medicine.

The suiting-up procedure is complicated. Rubber-soled booties must be tucked under a jumpsuit to ensure that spilled fluids don't drip into them. Purple latex gloves are layered underneath green ones; the different colours are used to ensure that rips in the outer layer are visible. Black N95 face masks pinch off the nostrils, forcing the wearer to breathe noisily through his or

$\checkmark$ her mouth. The staff call them Darth Vader masks. They also wear

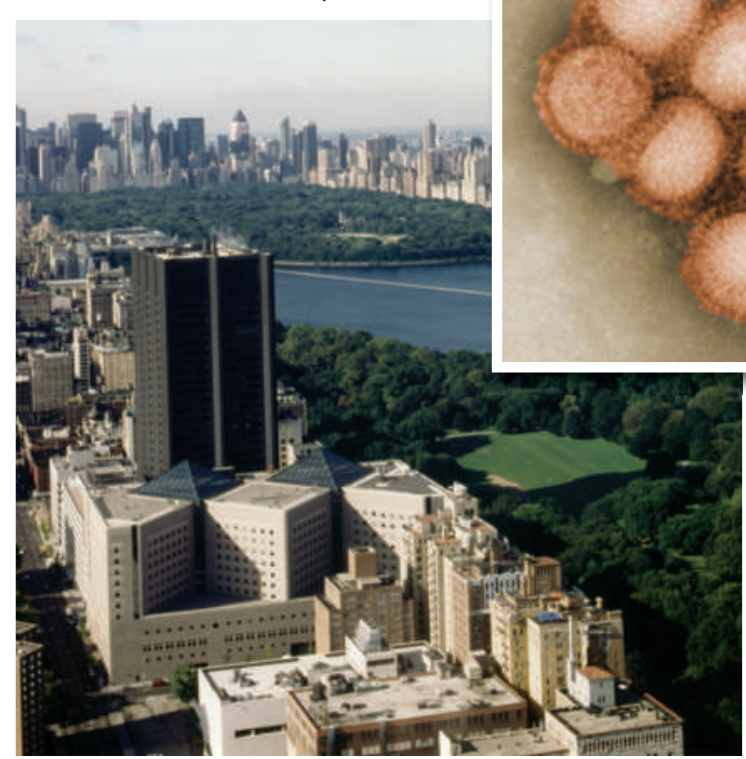

Biologists at the Mount Sinai School of Medicine are studying transmission of the pandemic $\mathrm{H} 1 \mathrm{~N} 1$ flu virus. papery mesh scrubs and white 'bunny suit' coveralls made from the material used to insulate houses. Biosafety officer Philip Hauck quotes Dante as he prepares to go in: "Abandon all hope ye who enter here."

In another preparatory room, they add heavy, belted, battery-powered air filters with a hose that hooks into the back of a white head-covering. Once it's adjusted, air begins rushing up over the face, puffing up the suit. The positive pressure in the suit, combined with the negative air pressure in the lab, ensures that the flow of air will carry any airborne pathogens away from the body.

This is the procedure that researchers at Mount Sinai go through every day to carry out their animal studies on deadly pathogens. Since May, pandemic H1N1 has been one of those pathogens. As the pandemic was emerging, John Steel and Anice Lowen secured strains of novel H1N1 from California and the Netherlands, and made plans to include them in their work modelling the transmission of influenza in guinea pigs. BSL-3 facilities are not required to work with H1N1, but Steel and Lowen's institutional review boards wanted the work to be carried out under the most stringent conditions on site.

Many labs use ferrets to model the transmission and pathology of human viruses, but the animals can be difficult to work with. Peter Palese, the head of microbiology at Mount Sinai, came up with the idea to work with guinea pigs a few years ago after reading an article published after the 1918 influenza pandemic, in which researchers in New Mexico noted that the infection had killed off a number of laboratory guinea pigs ${ }^{2}$. He convinced Lowen and Steel, assistant professors in his lab, to test whether the creatures would transmit human influenza. They did. In 2006, the group showed that the guinea pigs pass the virus between them with about the same efficiency as humans ${ }^{3}$, and the researchers still use them as a model of transmission 
even though their animals, purchased from a lab supplier, do not show symptoms or die from the disease.

The first thing the researchers did with the samples of pandemic $\mathrm{H} 1 \mathrm{~N} 1$ was to compare its transmissibility with that of seasonal flu. They squirted about 10,000 infectious H1N1 particles up the noses of four guinea pigs and, a day later, placed these animals in cages next to uninfected animals, separated by wire-mesh walls that allow respiratory droplets to pass through. Within five days all the exposed animals tested positive for the virus. The team's paper, published last month in the Journal of Virology, suggests that the H1N1 swine flu virus transmits just as efficiently as seasonal flu ${ }^{4}$. This is contrary to some work on transmission done at the start of the pandemic ${ }^{5}$ but mirrors real-world data showing that H1N1 spreads rapidly. "The pandemic sort of scooped us," says Lowen. Palese's group also found that previous exposure to the $\mathrm{H} 1 \mathrm{~N} 1$ and $\mathrm{H} 3 \mathrm{~N} 2$ subtypes of seasonal influenza limits the ability of exposed animals to become infected with the pandemic H1N1, which supports the idea that a seasonal flu infection or vaccine might offer some cross-protection against swine flu.

\section{What could it turn into?}

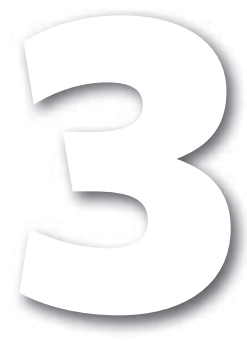

A deadly line-up of viruses is locked up in the computercontrolled safes at the Jean Mérieux/INSERM biosecurity level four (BSL-4) facility in Lyon, France, including Ebola, Nipah, Lassa, Hendra and Marburg. And in the next few weeks, scientists working there are planning to manufacture a new resident. They hope to test whether the highly transmissible pandemic H1N1 virus could reassort with its deadlier cousin, the $\mathrm{H} 5 \mathrm{~N} 1$ avian flu, to make a virus with the worst properties of both.

Classed as a national high-security facility, the laboratory is a three-storey shoebox made from armoured glass, perched above a biology research centre of INSERM, the $\sum$ national biomedical agency. The edifice is built to withstand earthquakes, bullets and explosives. It is also smack in the city centre, its entrance just metres from mothers pushing prams along the pavement on avenue Tony Garnier.

The lab itself is surrounded by empty corridors and a

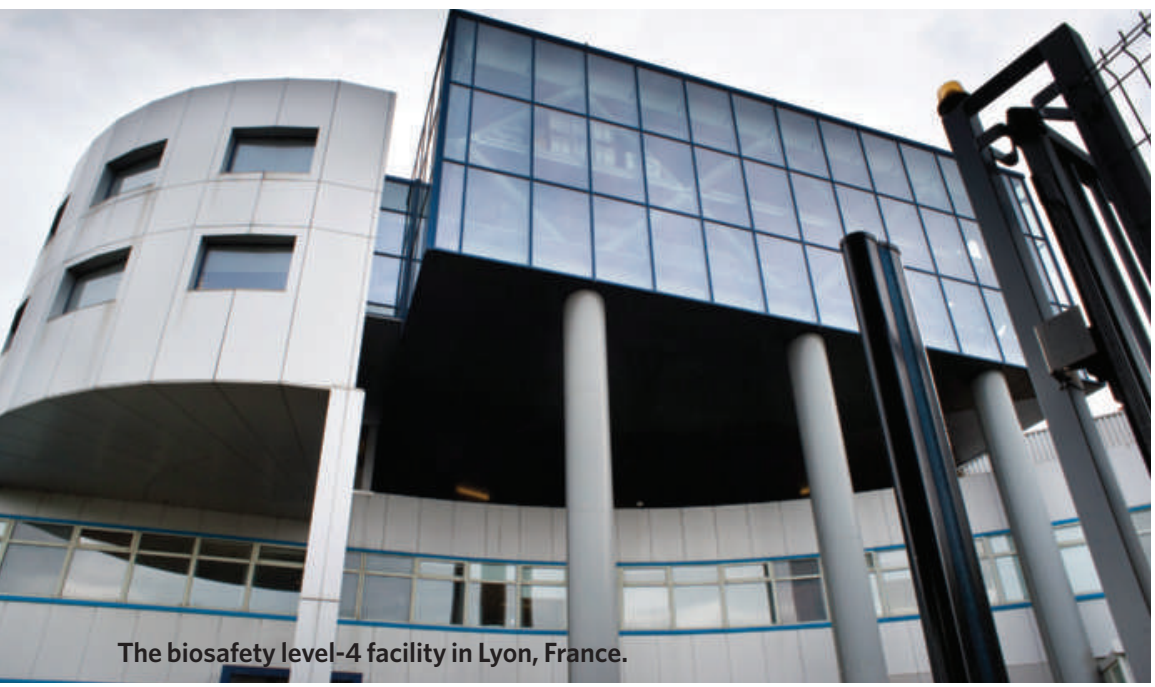

Inside the facility are the huge steel ferret cages that Steel and Lowen have adapted for their guinea-pig work so that two animals can fit inside each. They are now planning new experiments, hoping to find the sequences within the genome that enable the virus to transmit so well between humans compared with other swine-adapted influenza strains. They may create reassortments of the virus and then test how well they are transmitted by the guinea pigs. The results might aid in surveillance efforts aimed at identifying other strains from animals that could make the leap to humans.

Everything that goes into the BSL-3 lab must be decontaminated before coming out. This involves spraying down the bodysuit with ethanol, carefully peeling it off and then leaving the facility by way of a shower room. A pen and notepad won't survive the caustic chemical dunk by the exit, so the pages are scanned and put onto a CD that can be sprayed down.

Outside again on the Manhattan streets, H1N1 could be encountered in any cough or sneeze. Without all the protective gear, one feels rather vulnerable.

\section{Brendan Maher}

spartan decor of steel staircases, trusses, raised walkways and ventilation pipes. The air hums from the air-ultrafiltration and other support machinery that take up entire floors above and below. Under negative air pressure to stop viruses escaping in the event of a breach, the lab is split into three zones, with the airstream flowing towards the 'hottest' exposure risk zone, the animal house at the back.

When the current $\mathrm{H} 1 \mathrm{~N} 1$ pandemic began in April, priority lab time here was allocated to Bruno Lina, a virologist and flu researcher at the CNRS, France's basic-research agency, who works at the University of Claude Bernard Lyon-1. Pandemic $\mathrm{H} 1 \mathrm{~N} 1$ influenza is not itself a BSL-4 agent - a BSL-2 facility is adequate. But in France, the health ministry classes viral reassortment experiments of the sort Lina is performing as requiring BSL-4 precautions.

The ability to predict which reassortments might take place, and what type of flu viruses might result, could be key to predicting the behaviour of this and future pandemics. The eight genes in the influenza virus's segmented genome are easily swapped between strains. But to be viable, new gene combinations must also be able to work together to package themselves into a virus particle. Viruses within a subtype tend to reassort with one another more easily and generate more viable reassortants than do those from different subtypes.

Over the summer, Lina's team has been using the BSL-4 facility to investigate the likelihood that pandemic $\mathrm{H} 1 \mathrm{~N} 1$ will acquire resistance to the front-line antiviral drug oseltamivir (Tamiflu) through reassortment, and how easily these reassortants might spread. Resistance can emerge by spontaneous mutation, but given that seasonal H1N1 is already resistant to the drug and spreads easily, reassortment is perhaps the most likely way that pandemic H1N1 will acquire resistance - especially as seasonal H1NI and pandemic $\mathrm{H} 1 \mathrm{~N} 1$ are the same subtype. Since the start of the pandemic, Tamiflu-resistant strains have sporadically appeared in several countries but none has yet gained a foothold. That 


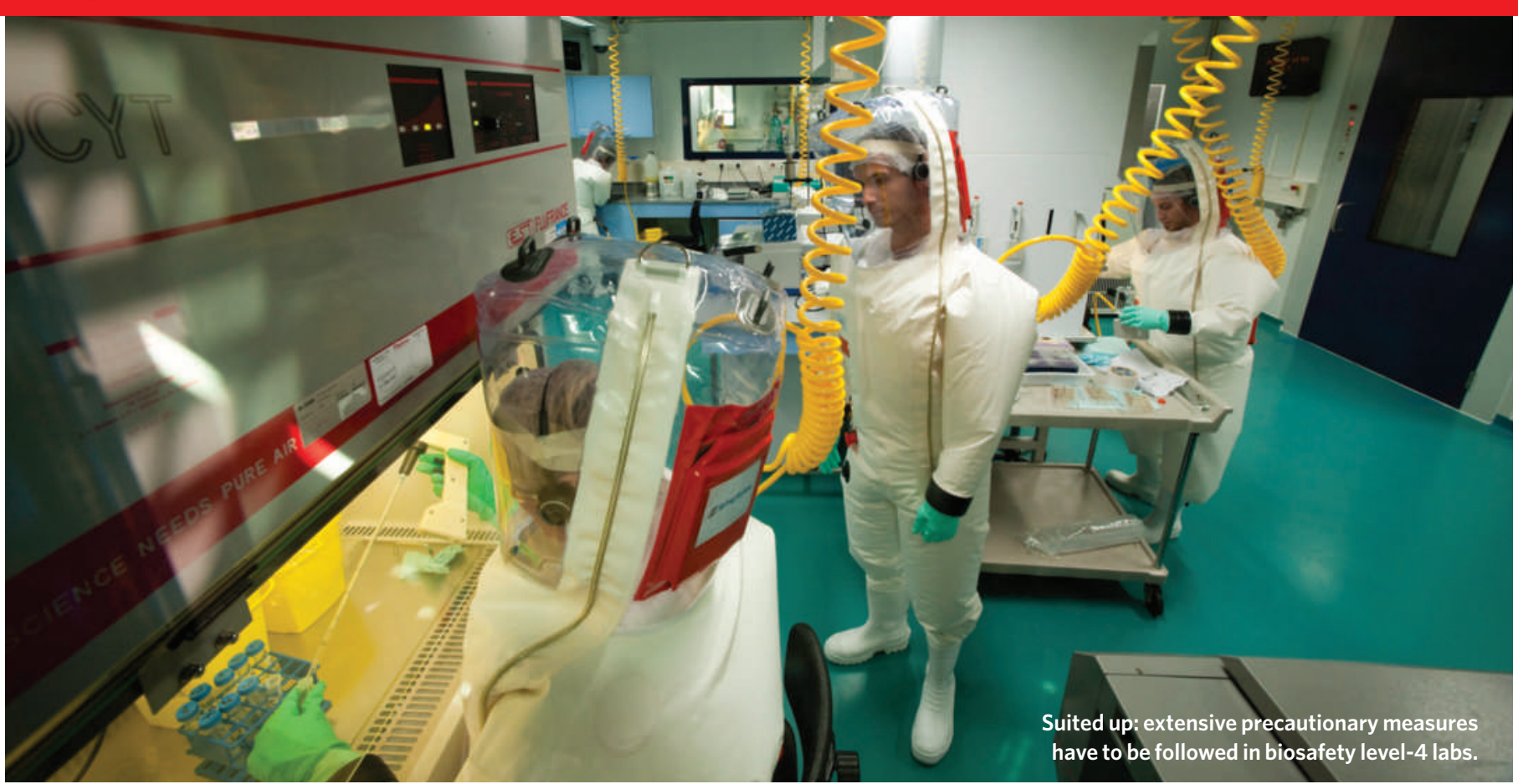

they haven't arisen more often or spread more easily may be because there is little seasonal H1N1 circulating, as pandemic $\mathrm{H} 1 \mathrm{~N} 1$ is outcompeting it - a large number of co-infections are needed for transmissible reassortants to arise.

In his work, Lina co-cultured the two $\mathrm{H} 1 \mathrm{~N} 1$ viruses in a cell line. He is now testing how pathogenic the reassortants are in mice, before using ferrets to test their capacity to spread. The biggest hazard that the scientists face in the lab is a bite or a scratch from an infected animal, so all manipulations are done on animals that have been anaesthetized remotely.

Lina is also preparing to submit a protocol to the facility's scientific board seeking the green light to try to reassort pandemic $\mathrm{H} 1 \mathrm{~N} 1$ and the H5N1 avian flu virus. "It's controversial research, but it is basic science that needs to be done," says Lina. H5N1 has killed more than half of the people it has infected since it resurged in 2003, but has rarely spread from one person to another. H1N1, on the other hand, seems to be as transmissible as seasonal flu, but mild in most people, if severe in some. The aim of Lina's proposal is to find out the probability of a reassortant arising that combines the lethality of H5N1 with the transmissibility of H1N1.

In particular, Lina is searching for putative molecular controls of the virus's segmented genome that may determine why some reassortants can package the virus and others can't. "We don't know which regions in $\mathrm{H} 5 \mathrm{~N} 1$ are responsible for that control," says Lina. That could have benefits for disease surveillance, he says. If researchers know the key genetic regions that facilitate reassortment, surveillance efforts could watch out for $\mathrm{H} 5 \mathrm{~N} 1$ or $\mathrm{H} 1 \mathrm{~N} 1$ viruses with changes in those regions, ones that might be on the verge of dangerous reassortments.

Lina will use reverse genetics to generate a soup of reassortants, test whether any are viable, and if they are, assess their virulence and transmissibility. Because H1N1 and $\mathrm{H} 5 \mathrm{~N} 1$ are different subtypes, Lina does not expect them to swap genes easily. In 2005, he tried to reassort H5N1 with seasonal H1N1 and H3N2 viruses, without success. "After a year we only had three reassortants, and none was fit," recalls Lina, "they just don't reassort well."

The experiments that Lina's team is carrying out in the BSL-4 lab aren't technically novel or difficult in themselves.
But the encumbrance of the safety procedures for getting in and out of the high-security lab make even the most straightforward procedures complicated, says Lina. Looking through the bulletproof window, one can see why. As the researchers move around the lab, they connect and disconnect their blindingly white spacesuits from some 60 yellow air hoses dangling from the ceiling. The hands of the researchers snipping away at tissue samples are wrapped in multiple layers of gloves to avoid accidental pricks. "It's like trying to do surgery wearing boxing gloves," says Hervé Raoul, director of the facility.

Before researchers can even begin to work in the lab, they must pass a three-week training course. It's only after a further 200 hours of practice that they are given any real autonomy in the lab, and they are never allowed inside alone. Users are limited to working a maximum of one four-hour shift a day, so that they are less likely to make mistakes. They are also encouraged to share any concerns about the behaviour of colleagues, or whether they themselves are going through a difficult patch, which might cause distrac-

- Bruno Lina tion and accidents.

Before Lina's experiments get approved, the facility's external scientific board will need to be convinced of the public-health justification, that the science is top-notch and that the experiments can be done safely, says Raoul. It will also need to be approved by government regulatory agencies. If all goes well, he could have his authorization in weeks, Lina says.

"Compared to the big guns in flu research, I'm a little guy," says Lina. "But having access on my doorstep to one of the rare BSL-4 facilities in the world with an animal house is a big advantage."

\section{Declan Butler}

1. Childs, R. A. et al. Nature Biotechnol. 27, 797-799 (2009).

2. Lamb, F. H. \& Brannin, E. B. J. Am. Med. Assoc. 72, 1056-1062 (1919).

3. Lowen, A. C., Mubareka, S., Tumpey, T. M., García-Sastre, A. \& Palese, P. Proc. Natl Acad. Sci. USA 103, 9988-9992 (2006)

4. Steel, J. et al. J. Virol. doi:10.1128/JVI.01732-09 (2009).

5. Maines, T. R. et al. Science 325, 484-487 (2009).

\section{See Editorial, page 137, and News, page 146}

